\title{
GELIFICACIÓN IÓNICA DE PECTINA DE BAJO GRADO DE ESTERIFICACIÓN EXTRAIIDA DE MANZANAS INMADURAS DE RALEO
}

\author{
IONIC GELATION OF LOW-ESTERIFICATION DEGREE PECTINS \\ FROM IMMATURE THINNED APPLES
}

\section{Agustín Rascón-Chu, Ana-Luisa Martínez-López², Elizabeth Carvajal-Millán², Karla G. Martínez-Robinson ${ }^{2}$ y Alma C. Campa-Mada ${ }^{2}$}

\begin{abstract}
'Laboratorio de Biotecnología de Plantas y Hongos, Coordinación de Tecnología de Alimentos de Origen Vegetal (CTAOV). ${ }^{2}$ Laboratorio de Biopolímeros, CTAOA, Centro de Investigación en Alimentación y Desarrollo, AC. Km. 0.6 Car. a La Victoria. 83304, Hermosillo, Sonora, México. Tel. (662) 289-2400, Fax (662) 280-0422.

*Autor para correspondencia (arascon@ciad.mx)
\end{abstract}

\section{RESUMEN}

El raleo de frutos es una práctica común en los huertos de manzana (Malus $x$ domestica Borkh.) para obtener frutos de mayor tamaño y promover el retorno de la floración. Esta práctica genera manzanas inmaduras y de diámetro pequeño $(10$ a $40 \mathrm{~mm})$ que son utilizadas ocasionalmente como alimento para ganado. La utilización de la manzana de raleo como fuente alternativa de pectina podría incrementar la rentabilidad de los huertos y el desarrollo de nuevos procesos de transformación. La pectina es un polisacárido localizado en la pared celular de los tejidos vegetales, que se ha extraído de distintas fuentes y utilizado exitosamente en la industria alimentaria. Este trabajo de investigación tuvo como objetivo extraer la pectina de manzanas de raleo de las variedades Golden Delicious (GD) y RedChief Delicious (RChD), y determinar las propiedades gelificantes del polisacárido. La pectina fue extraída mediante hidrólisis ácida controlada, con un rendimiento de 12 y $11 \%$ (p/p) para GD y RChD, respectivamente. Las pectinas obtenidas fueron desesterificadas por vía alcalina y presentaron grados de esterificación de 35 $\%(\mathrm{GD})$ y $42 \%$ (RChD). Ambas pectinas formaron geles iónicos inducidos por calcio, con una elasticidad de 147 y $90 \mathrm{~Pa}$ para GD y RChD, respectivamente, a una concentración de polisacárido de $2 \%(\mathrm{p} / \mathrm{v})$. La pectina desesterificada de manzanas inmaduras de raleo presenta propiedades físico-químicas y gelificantes que podrían ser de interés para las industrias alimentaria, farmacéutica y cosmética.

Palabras clave: Malus x domestica, pectina baja en metoxilo, geles iónicos, calcio, reología.

\section{SUMMARY}

Fruit thinning is a regular practice in apple (Malus $x$ domestica Borkh.) orchards to increase fruit size and to promote blooming. This practice generates immature, small (10 to $40 \mathrm{~mm}$ diameter) fruits, which are occasionally used as cattle feed. The use of thinned apples for pectin extraction could increase orchards profitability and promote development of new transformation processes. Pectin is a polysaccharide located on the cell wall of plant tissues. Commercial pectins are extracted from different plant sources and have been mainly used in the food industry. The present research aimed to extract pectin from thinned Golden Delicious (GD) and RedChief Delicious (RChD) immature fruits and determine the effect of de-esterification on pectin gelling properties. Pectin was extracted using a controlled acid hydrolysis. Extraction yields were 12 and $11 \%(\mathrm{w} / \mathrm{w})$ for $\mathrm{GD}$ and $\mathrm{RChD}$, respectively. De-esterified pectins were obtained by alkaline hydrolysis and showed an esterification degree of 35 and $42 \%$ for GD and RChD, respectively. Using a polysaccharide concentration of $2 \%(w / v)$, both de-esterified pectins formed ionic gels induced by calcium, which presented an elasticity of 147 and $90 \mathrm{~Pa}$ for GD and RChD, respectively.
In short, thinned GD and RChD immature fruits are a source of pectin with yield and properties similar to commercially available pectins.

Index words: Malus $x$ domestica, low methoxy pectin, ionic gels, calcium, rheology.

\section{INTRODUCCIÓN}

Características de los polisacáridos como estructura, conformación e interacción molecular están estrechamente vinculadas con sus propiedades de solubilidad, viscosidad y capacidad de gelificar. Esta relación estructura-propiedades ha incrementado el interés de modificar algunos polisacáridos para el desarrollo y mejoramiento de las propiedades funcionales de los mismos (Carvajal-Millan et al., 2005). La pectina es un polisacárido ácido presente en las paredes celulares de las plantas, principalmente en dicotiledóneas (15\%) (Ishii, 1997), que se utiliza principalmente como agente texturizante en las industrias alimentaria, farmacéutica y de cosméticos.

La pectina está constituida de una cadena lineal predominantemente de a-(1-4)-D-ácido galacturónico con residuos de azúcares neutros unidos que forman parte de la cadena principal. Los residuos de ácido galacturónico se encuentran frecuentemente esterificados por grupos metilo en C2 o C3 (Yoo et al., 2006).

De acuerdo al grado de esterificación (GE) las pectinas se clasifican en alto GE (> 50 \%) y bajo GE (<50\%) (Rinaldo, 1996). Las pectinas de alto GE gelifican bajo condiciones ácidas, mientras que las de bajo GE forman geles iónicos (Rinaldo, 1996). El calcio induce la gelificación iónica de las pectinas de bajo GE mediante el acoplamiento de cadenas que forman un modelo llamado caja de huevo (Braccini y Pérez, 2001; Powell et al., 1982). La habilidad de las pectinas para interaccionar con cationes bivalentes, principalmente calcio, es de suma importancia en sus propiedades funcionales (Costa et al., 2008; Renard y Jarvis, 1999). 
Las pectinas son extraídas principalmente de la cáscara de cítricos (Citrus spp.) y de la pulpa de la manzana (Malus $x$ domestica Borkh.), entre otros frutos. Sin embargo, la recuperación de la pectina a partir de sub-productos agrícolas permitiría generar un uso alternativo de materiales de desecho ricos en compuestos funcionales como pectinas (Mollea et al., 2008). Las manzanas de raleo son un subproducto agrícola que se genera durante el manejo de los huertos. En algunos casos este subproducto es utilizado como alimento para ganado o como materia prima para el composteo sólido. En el presente estudio se extrajo la pectina de manzanas de raleo de las vars. Golden Delicious y RedChief Delicious, y se investigó el efecto de desesterificar en las propiedades gelificantes del polisacárido.

\section{MATERIALES Y MÉTODOS}

\section{Materiales}

Las manzanas de raleo fueron obtenidas de un huerto comercial localizado en las coordenadas $28^{\circ} 24^{\prime} 18^{\prime \prime}$ N, $106^{\circ} 52^{\prime}$ O" W, a una altura promedio de 2060 msnm, en el Estado de Chihuahua, México. Esta zona es de clima semiseco templado, con veranos de temperatura templada, inviernos fríos con descensos de temperaturas por debajo de los $-10^{\circ} \mathrm{C}$. La temperatura media anual varía de 10 a $14{ }^{\circ} \mathrm{C}$, y la precipitación media anual registra de 500 a 600 mm, con una a tres nevadas esporádicas en invierno. Las muestras se tomaron de las zonas del huerto donde se estaba llevando a cabo el aclareo de los manzanos, consistentes en $6 \mathrm{~kg}$ de frutos de 20 a $30 \mathrm{~mm}$ de diámetro, de cada variedad. Los frutos muestreados se congelaron a una temperatura de $-20^{\circ} \mathrm{C}$, para su posterior liofilización. Los frutos liofilizados se sometieron a un proceso de molienda, primero en un molino eléctrico de laboratorio (Thomas Wiley® Mini-Mill, Modelo 3383-L10. USA) con criba de $2 \mathrm{~mm}$, y finalmente en un molino Cyclotec ${ }^{\circledR}$ (Cyclotec Sample Mill, Modelo 1093. Sweden) con una criba de $1 \mathrm{~mm}$. Las muestras liofilizadas y molidas se almacenaron en bolsas de polietileno herméticas a temperatura ambiente.

\section{Extracción}

La extracción de la pectina se realizó mediante la metodología reportada por Rascón-Chu et al. (2009). Los extractos de pectina obtenidos de ambas variedades fueron purificados. Para cada variedad de manzana se suspendieron $10 \mathrm{~g}$ de la pectina extraída en $1000 \mathrm{~mL}$ de solución de $\mathrm{NaCl}$ 0.1 N. Las suspensiones de pectina se sometieron a centrifugación $\left(12,096 \mathrm{~g}, 20^{\circ} \mathrm{C}, 10 \mathrm{~min}\right)$. El sobrenadante fue dializado (poro $12 \mathrm{Da}, 24$ h, $4^{\circ} \mathrm{C}$ ) para la eliminación de monosacáridos, oligosacáridos y otros componentes de bajo peso molecular. El dializado fue precipitado con etanol a $65 \%(\mathrm{v} / \mathrm{v})$ por $4 \mathrm{~h}$ a $4{ }^{\circ} \mathrm{C}$. El precipitado fue recuperado y secado con intercambio de disolventes, primero en etanol a $80 \%$ (v/v), luego en etanol absoluto y finalmente en acetona, la cual se evaporó para obtener la pectina seca.

\section{Desesterificación química}

La pectina fue desesterificada (PDE) por tratamiento alcalino a un $\mathrm{pH}$ de 10.5 , a $4{ }^{\circ} \mathrm{C}$ durante $10 \mathrm{~min}$. La reacción se detuvo con la disminución del $\mathrm{pH}$ a 4 . El extracto fue dializado (poro $12 \mathrm{Da}, 24 \mathrm{~h}, 4^{\circ} \mathrm{C}$ ) y la solución fue precipitada con etanol a $65 \%(\mathrm{v} / \mathrm{v})$ por $4 \mathrm{~h}$ a $4{ }^{\circ} \mathrm{C}$. El precipitado fue recuperado y secado con intercambio de disolventes; específicamente, etanol a $80 \%$ (v/v), seguido por etanol absoluto y finalmente acetona para recuperar la pectina desesterificada en polvo.

\section{Determinación del grado de esterificación (GE)}

El grado de esterificación (GE) de las pectinas sin tratar y tratadas (PDE) con álcali fue determinado por espectrofotometría de infrarrojo transformada de Fourier (Nicolet Protégé 460® Spectrometer. USA). El espectro obtenido fue comparable en su perfil al de un espectro de pectina comercial. Para determinar el GE se utilizó la relación del área de la banda correspondiente a los grupos carboxilos y el área de los grupos esterificados, como se indica en la ecuación reportada por Monsoor et al. (2001), y UriasOrona et al. (2010).

GE = [área de grupo carboxilo esterificado / (área de grupo carboxilos esterificados + área grupos carboxilos no esterificado)] x100

\section{Caracterización de la pectina desesterificada (PDE)}

Contenido de azúcares. La composición de azúcares de la PDE fue determinada a partir de una hidrólisis con ácido trifluoracético $4 \mathrm{~N}$ a $120{ }^{\circ} \mathrm{C}$ durante $2 \mathrm{~h}$. La reacción se detuvo a las $4 \mathrm{~h}$ en cama de hielo y los extractos fueron evaporados con un flujo constante de aire a $40^{\circ} \mathrm{C}$ en un sistema de evaporación con temperatura y flujo de gases controlado (Techne®, DB-3A. UK). Los extractos evaporados fueron solubilizados en $500 \mu \mathrm{L}$ de agua destilada. Se utilizó inositol como estándar interno. Las muestras fueron filtradas a través de un filtro con tamaño de poro de 0.45 $\mathrm{mm}$ (Whatman) y analizadas por cromatrografía líquida de alta resolución (CLAR) acoplado a un detector de índice de refracción Star 9040® (Varian, St. Helens. Australia). Se usó una columna SupelcoPb® $(300 \times 7.8$ mm; Supelco, Inc. Bellefonte, PA, USA), dluída con agua grado CLAR a un flujo de $0.6 \mathrm{~mL} \mathrm{~min}^{-1}$ a $80^{\circ} \mathrm{C}$. Mediante el programa CWSC (Chromatography Workstation System Control) versión 5.50, se realizó el análisis de estándares de azúcares y el cálculo de la concentración en las muestras mediante 
curvas patrón, de acuerdo con metodología previamente reportada (Carvajal-Millan et al., 2005).

Contenido de proteínas. Se determinó en la PDE de acuerdo con el método de Bradford (Bradford, 1976). Este método emplea el colorante hidrofóbico azul de Coomassie G-250, el cual al encontrarse en el entorno hidrofóbico del interior de una proteína, origina un color azul. Mediante técnicas espectrofotométricas (Varian, Cary 1E UV-VIS® Spectrophotometer. Australia) es posible determinar la absorbancia a $595 \mathrm{~nm}$. El valor de absorbancia a esa longitud de onda se utilizó para inferir la concentración presente mediante interpolación en una curva de calibración. La proteína patrón utilizada para dicha curva fue seroalbúmina bovina.

Fenoles totales. El contenido de fenoles totales se determinó espectrofotométricamente según el método FolinCiocalteu reportado por Cindrić et al. (2011) para manzana. Brevemente, $0.5 \mathrm{~mL}$ de la muestra se pipetearon en un matraz aforado de $10 \mathrm{~mL}$ que contenía $0.5 \mathrm{~mL}$ de reactivo de Folin-Ciocalteu, $5 \mathrm{~mL}$ de agua destilada y $1.5 \mathrm{~mL}$ de $\mathrm{Na}$ ${ }_{2} \mathrm{CO}_{3}$ a $20 \%$. Durante la oxidación de compuestos fenólicos, ácido fosfomolíbdico y fosfotúngstico, contenidos en el reactivo de Folin- Ciocalteu, se redujeron a óxidos de molibdeno y tungsteno de color azul. Después de 2 h se midió la absorbancia (Varian, Cary 1E UV-VIS® Spectrophotometer, Australia) de coloración azul a $\lambda=765 \mathrm{~nm}$, contra un blanco de muestra. Las mediciones se compararon con una curva estándar de soluciones de ácido gálico y se expresaron en gramos de equivalentes de ácido gálico por 100 $\mathrm{g}$ de muestra $\pm \mathrm{DE}$. Todas las mediciones se hicieron por triplicado.

Viscosidad intrínseca. La viscosidad específica $\left(\eta_{\mathrm{sp}}\right)$ de la PDE en solución fue medida con un viscosímetro capilar AVS 400® (Schott Geräte, Hofheim, Germany) equipado con un tubo capilar de Oswald. La viscosidad intrínseca [n] fue determinada por el método de Kraemer modificado por Mead y Fouss (Mead y Fouss, 1942), reportado por Rascón-Chu et al. (2009).

Peso molecular. El peso molecular viscosimétrico (Mv) se calculó a partir de la relación de Mark-Houwink, expresada como $M_{v}=([\eta] / k)^{1 / \alpha}$, donde las constantes $k$ y a son 0.0436 y 0.78 , respectivamente, según los valores reportados por Urias-Orona et al. (2010).

Propiedades viscoelásticas. Estas propiedades de la PDE fueron estudiadas mediante mediciones de los módulos de almacenamiento $\left(G^{\prime}\right)$ y pérdida $\left(G^{\prime \prime}\right)$, con $5 \%$ de deformación y una frecuencia de $0.25 \mathrm{~Hz}$ a $25^{\circ} \mathrm{C}$, en un reó- metro de baja deformación (AR-1500ex®, TA Instruments USA) en modo oscilatorio. La geometría utilizada fue de plato (50 mm de diámetro). Las pectinas fueron disueltas con agitación vigorosa en agua desionizada. Se trabajó en un intervalo de $\mathrm{pH}$ de 3 a 11. Los geles iónicos se formaron con uso de $\mathrm{CaCl}_{2}$. La concentración del ion bivalente fue determinada mediante una curva de firmeza con respecto a la concentración, en un texturómetro (TA.XT2®, TTC. USA) equipado con el programa XTRAD versión 3.7. Los geles fueron deformados por compresión a una velocidad constante de $1.0 \mathrm{~mm} \mathrm{~s}^{-1}$ a una distancia de $4 \mathrm{~mm}$ de la superficie del gel con un émbolo cilíndrico (diámetro $25.4 \mathrm{~mm}$ ). La altura del pico a los $4 \mathrm{~mm}$ de compresión se denominó dureza del gel (Carvajal-Millan et al., 2005). La concentración de PDE fue 2 \% (p/v).

\section{Análisis estadísticos}

Para el análisis de datos de cada variedad se utilizó un análisis de varianza conforme a un diseño completamente al azar con tres repeticiones. La comparación y agrupamiento de medias se hicieron por medio de la prueba de Tukey $(a<0.05)$. Estos análisis se llevaron a cabo con el paquete estadístico SAS versión 6.08 (SAS Institute, 1994).

\section{RESULTADOS Y DISCUSIÓN}

\section{Extracción y caracterización de pectina}

Entre las variedades GD y RChD no hubo diferencias significativas $(a<0.05)$ en la recuperación de pectina obtenida de manzanas de raleo, con rendimientos promedio de 12 y $11 \%$ p/p, respectivamente (Cuadro 1), comparables al antes reportado en frutos maduros de la variedad GD con un valor de 16 \% (p/p) (Rascón-Chu et al., 2009). El grado de esterificación de las pectinas extraídas de GD y RChD (antes de la desesterificación) fue 57 y 63 \%, respectivamente, que las califica como pectinas de alto grado de esterificación según el criterio reportado por May (1997), quien apuntó que valores de esterificación por encima de 50 \% se consideran de alto grado (Figuras 1a y b).

En pectina de manzana extraída por métodos ácidos similares (Marcon et al., 2005; Rascón-Chu et al., 2009) se han reportado grados de esterificación de hasta $69 \%$. Generalmente es conocido que las condiciones de extracción alteran la estructura de las pectinas nativas al modificar la estructura de la pared celular. En este contexto, la similitud de las pectinas de manzana de raleo con pectinas de manzanas en madurez comercial, sugiere sólidamente que las manzanas inmaduras pueden ser una fuente potencial de este polisacárido. 
Cuadro 1. Rendimiento y características físico-químicas de la pectina extraída de manzanas inmaduras de raleo, en dos variedades.

\begin{tabular}{lcc}
\hline & Golden Delicious & RedChief Delicious \\
\hline Rendimiento $(\%, \mathrm{p} / \mathrm{p})$ & $12 \mathrm{a}$ & $11 \mathrm{a}$ \\
{$[\mathrm{n}](\mathrm{mL} / \mathrm{g})$} & $439 \mathrm{a}$ & $362 \mathrm{~b}$ \\
Peso molecular $(\mathrm{kDa})$ & $173 \mathrm{a}$ & $129 \mathrm{~b}$ \\
$\mathrm{GE}(\%)$ & $57 \mathrm{~b}$ & $63 \mathrm{a}$ \\
$\mathrm{GE}(\%)$ después de desesterificación & $35 \mathrm{~b}$ & $42 \mathrm{a}$ \\
\hline
\end{tabular}

Medias con la misma letra en cada fila son estadísticamente iguales (Tukey, 0.05). GE = grado de esterificación.
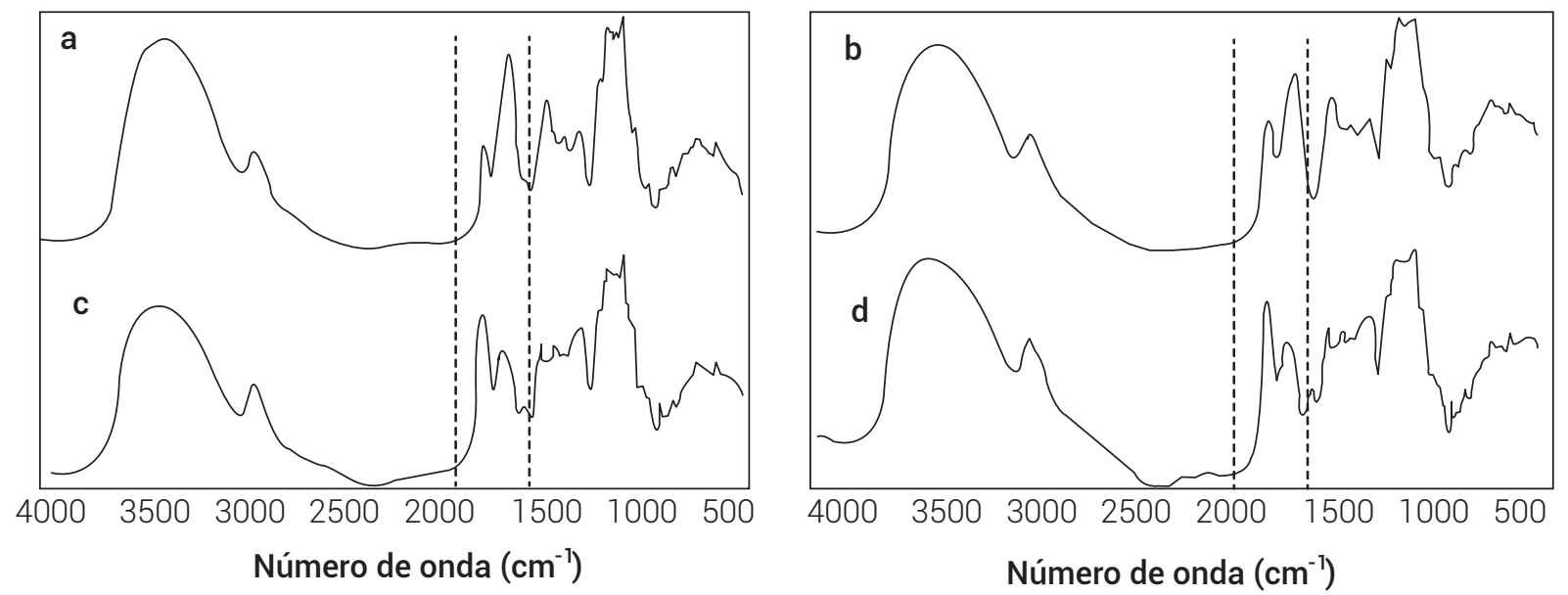

Figura 1. Espectros infrarrojos con Transformada de Fourier de pectina proveniente de frutos de manzanas de raleo de las vars. (a) Golden Delicious y (b) RedChief Delicious, extraídas por hidrólisis ácida controlada; (c) Golden Delicious; y (d) RedChief Delicious después de la desesterificación alcalina, respectivamente.

En las Figuras 1c y $1 d$ se muestran los perfiles de absorción en el infrarrojo de las pectinas antes mencionadas, después de haber sido desesterificadas (PDE) por tratamiento alcalino. En las pectinas PDE se observó una disminución en la intensidad de las bandas de absorción ubicadas en el rango de los 1600 a $1800 \mathrm{~cm}^{-1}$. El porcentaje de esterificación de la pectina puede estimarse con base en el cociente entre el área del pico a $1753 \mathrm{~cm}^{-1}$ (promedio) y la suma de las áreas de los dos picos, 1753 y $1632 \mathrm{~cm}^{-1}$, según demostraron Monsoor et al. (2001). Así, las PDE presentaron una disminución del grado de esterificación ya que rindieron valores de 35 y $42 \%$ para las vars. GD y RChD (Cuadro1), y corresponden a los espectros de fenoles totales (FT-IR) mostrados en las Figuras 1c y $1 \mathrm{~d}$. En contraste, Ström y Williams (2003) reportaron un valor de esterificación de $49 \%$ en pectinas cítricas desesterificadas con el mismo método.

La viscosidad intrínseca [n] y el peso molecular para PDE de GD y RChD muestran diferencias significativas entre ambas variedades, ya que GD presenta valores más altos que RChD. Lo anterior confirma el efecto genotípico sobre las propiedades fisicoquímicas del polisacárido que se ex- trae (Fishman et al., 1991), posiblemente por diferencias a nivel de pared celular. Una de las metas de este trabajo era conocer la naturaleza del polisacárido que se obtendría de los frutos muy inmaduros, ya que se desconocía si alcanzarían a presentar un perfil típico de las pectinas comerciales.

En la pectina aquí obtenida el azúcar mayoritario fue el ácido galacturónico, aunque también se encontraron residuos de azúcares neutros como galactosa, xilosa, ramnosa, arabinosa y glucosa (Cuadro 2). El hecho de que el ácido galacturónico sea el azúcar mayoritario confirma que nuestro polisacárido se ubica dentro del grupo de las pectinas. Además, el contenido en azúcares neutros concuerda con una pectina con presencia de RG I y RG I| en su estructura.

El contenido de fenoles encontrados en la pectina puede deberse a una co-extracción, ya que los presentes resultados no demuestran que dichas estructuras formen parte de la molécula de pectina, como es el caso para pectinas de remolacha (Beta vulgaris), con ácido ferúlico en posición 0-2 arabinosa, o bien 0-6 galactosa en RG I 


\begin{tabular}{|c|c|c|}
\hline Compuesto & Golden Delicious & RedChief Delicious \\
\hline \multicolumn{3}{|l|}{ Carbohidratos } \\
\hline Ácido galacturónico & $57.34 \mathrm{a}$ & $50.90 \mathrm{~b}$ \\
\hline Galactosa & $34.04 \mathrm{a}$ & $29.66 b$ \\
\hline Arabinosa & $3.01 \mathrm{a}$ & $7.56 b$ \\
\hline Xilosa & $0.31 b$ & $1.79 a$ \\
\hline Glucosa & $1.49 b$ & $3.23 \mathrm{a}$ \\
\hline Ramnosa & $2.61 \mathrm{~b}$ & 5.39 a \\
\hline Proteína & $0.033 b$ & $0.039 a$ \\
\hline Fenoles Totales & $0.947 \mathrm{~b}$ & $1.23 \mathrm{a}$ \\
\hline
\end{tabular}

Entre filas, las medias con la misma letra son estadísticamente iguales (Tukey, 0.05).

(Saulnier y Thibault, 1999). Bunzel et al. (2009) reportaron la caracterización de pectinas feruladas en productos de Amaranthus caudatus, pero en el presente estudio no se encontró evidencia de que los fenoles formen parte de la estructura de la pectina de frutos de manzana en etapas iniciales de crecimiento, aunque los compuestos fenólicos se encuentran en todo el fruto, contribuyen al color y a la capacidad antioxidante de las manzanas (Francini y Sebastiani, 2013).

El contenido de proteína (0.033 g/100 g de pectina) se encuentra dentro del promedio para otros cultivares de manzana sujetos al mismo método de extracción (Einhorn-Stoll et al., 2007). Esta fracción de proteína puede contribuir en gran medida a la capacidad emulsificante, como en el caso de pectinas de B. vulgaris cuando su presencia es más importante (Siew y Williams, 2008).

\section{Propiedades viscoelásticas}

El comportamiento del módulo de pérdida (G") para ambas pectinas en solución a $2 \%(\mathrm{p} / \mathrm{v})$ mediante reología dinámica de baja deformación, se muestra en la Figura 2. Conforme se incrementó el pH de la solución, el valor de G" de las soluciones disminuyó porque las pectinas tienen naturaleza polielectrolítica. A valores de $\mathrm{pH}$ neutro y básico (i.e., con los grupos carboxilos predominantemente ionizados), las fuerzas electrostáticas de repulsión son dominantes, lo que provoca enrollamiento aleatorio de las cadenas de las pectinas en solución. Sin embargo, a pH bajo (i.e., con un bajo grado de ionización), el comportamiento reológico cambia notablemente, pues provoca el estiramiento de las cadenas y por consiguiente incrementa la viscosidad (Lootens et al., 2003). A valores de pH 3, 5 y 7 , las variedades presentan diferencias significativas. En estos valores de $\mathrm{pH}$ se refleja claramente el efecto sobre la ionización de los carboxilos libres debidos a la desesteri- ficación inicial, y el comportamiento observado está relacionado directamente con sus diferencias en el grado de esterificación (GE).

En contraparte, los valores de G" entre las variedades no son significativamente diferentes $(a<0.05)$. Lo anterior podría explicarse por una alteración subsecuente de la estructura en un medio tan alcalino, capaz de desesterificar aún más las pectinas hasta igualar su capacidad de asociación intermolecular. Esta última hipótesis podría explicar también la magnitud tan pequeña de G". En las aplicaciones para la industria alimentaria, los alimentos procesados en los cuales estas pectinas pueden utilizarse, con frecuencia presentan valores de $\mathrm{pH}$ entre 5 y 7 , por lo que el comportamiento aquí observado para G" resulta conveniente.

Según el análisis de reología de alta deformación de los geles de pectinas desesterificadas (PDE) en ambas variedades, formados a diferentes concentraciones de calcio (Figura 3), el calcio interactúa de manera iónica con los grupos carboxílicos del ácido galacturónico que forman las cadenas de PDE, como reportaron Braccini y Pérez (2001). En función de la concentración de calcio usada para la formación de gel, la firmeza del gel de PDE en ambos cultivares aumentó conforme la concentración de calcio utilizada incrementó de 0.2 hasta $0.8 \%$. Sin embargo, a una concentración mayor de calcio $(1.0 \%$ p/v) la firmeza del gel disminuyó ligeramente, lo cual podría estar relacionado con el hecho de que una alta concentración de calcio puede incrementar la fuerza iónica de la solución e influir así en la conformación de la cadena al disminuir la participación de puentes de hidrógeno durante la formación del gel (Capel et al., 2006; Ström y Williams, 2003).

El gel de PDE de la var. GD presenta menor fuerza que el gel de PDE de la var. RChD, lo cual puede explicarse por una 


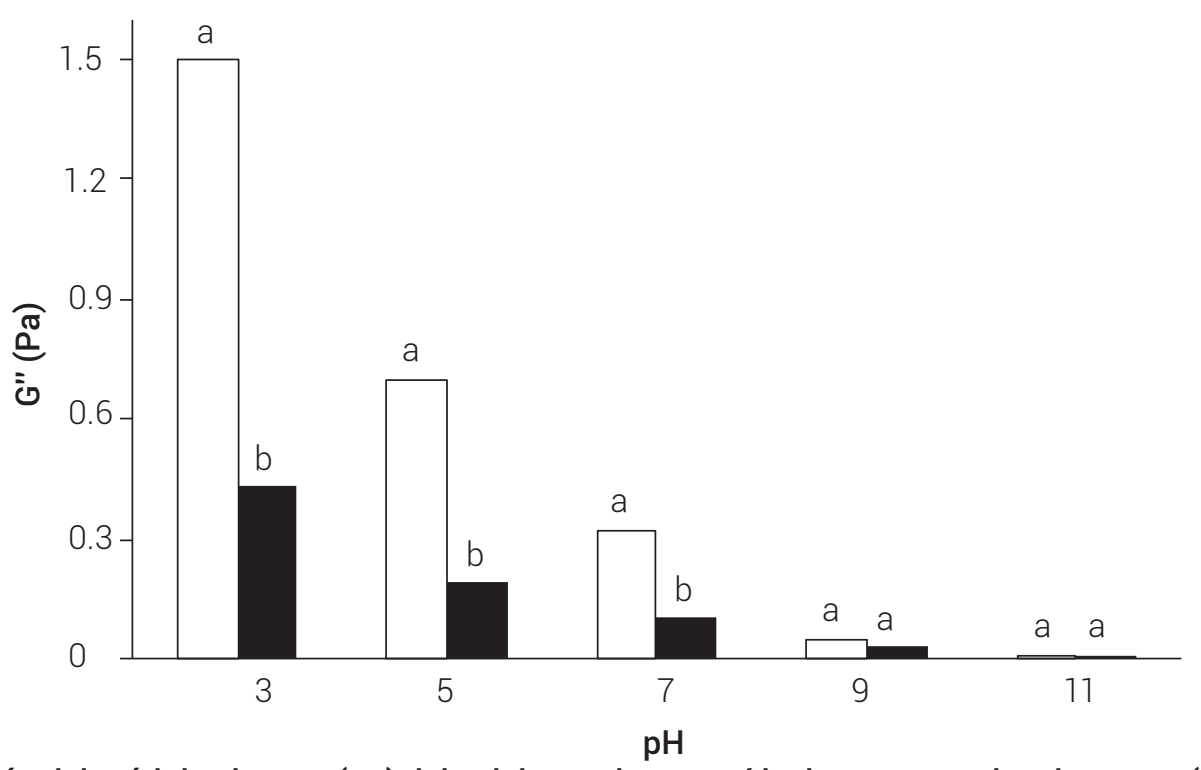

Figura 2. Comparación del módulo viscoso (G") del gel de pectina extraída de manzana de raleo a $2 \%(\mathrm{p} / \mathrm{v})$, de las variedades Golden Delicious ( $\square$ ) y RedChief Delicious $(\square)$ y a distintos valores de $\mathrm{pH}$, entrecruzados con $\mathrm{CaCl}_{2}$ a $0.8 \%(\mathrm{p} / \mathrm{v})$. Para un mismo valor de $\mathrm{pH}$, las medias con la misma letra son estadísticamente iguales (Tukey, 0.05).

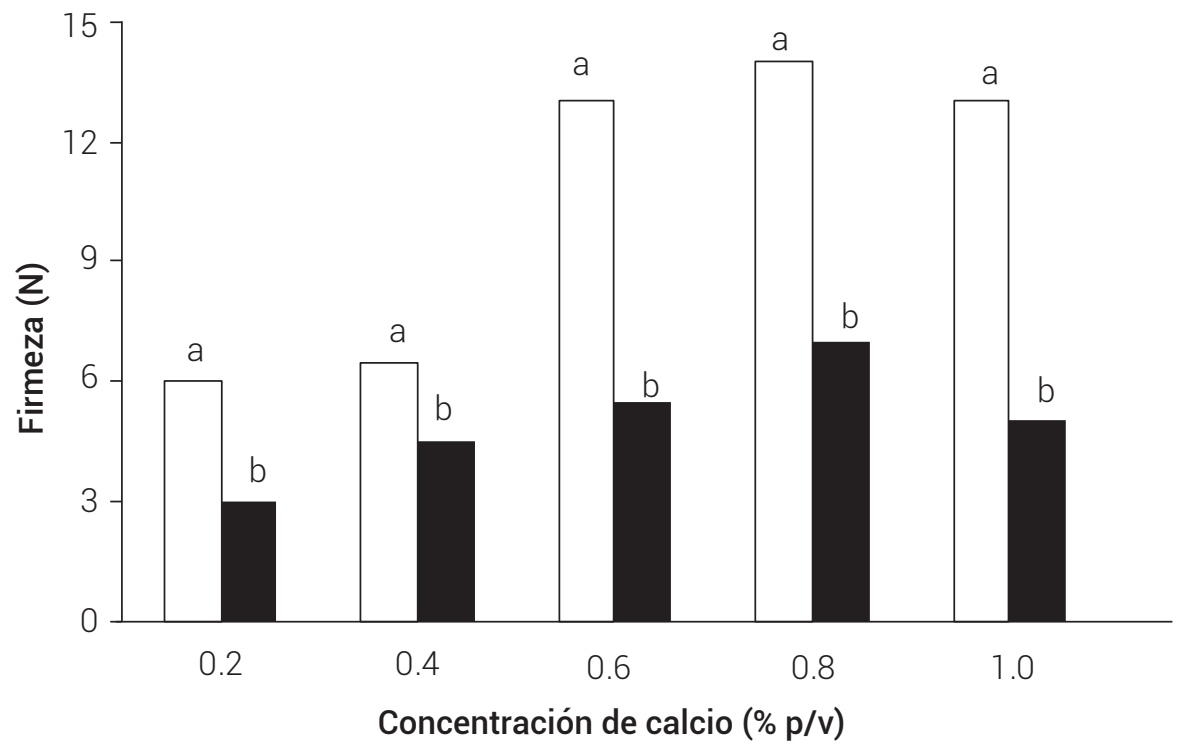

Figura 3. Comparación de la firmeza del gel de pectina extraída de manzana de raleo a $2 \%(\mathrm{p} / \mathrm{v})$, de las variedades Golden Delicious ( $\square$ ) y RedChief Delicious ( $\square$ ), a distintas concentraciones de calcio. Para un mismo valor de concentración de calcio, las medias con la misma letra son estadísticamente iguales (Tukey, 0.05).

menor presencia de grupos carboxilos libres (mayor en GE), además de presentar un menor peso molecular (Cuadro 1). Si bien el análisis que se realizó fue para comparar las dos variedades, no escapa a nuestra atención un comportamiento particular en la firmeza de la pectina de RChD, que se eleva considerablemente con valores de $0.6 \%$ o más de $\mathrm{CaCl}_{2}$. Aparentemente, la interacción con calcio puede llegar a un nivel de equilibrio, según los carboxilos liberados por la desesterificación, más allá del cual la firmeza no puede seguir incrementándose, y la acumulación de sales de calcio actúa sobre el equilibrio del sistema. Será necesario efectuar más investigación en este último supuesto para poder confirmar nuestra explicación.

La influencia del pH en el módulo de almacenamiento $\left(\mathrm{G}^{\prime}\right)$ de los geles de pectina a $2 \%(\mathrm{p} / \mathrm{v})$, con calcio a $0.8 \%$ 
$(\mathrm{p} / \mathrm{v})$ como agente gelificante, se muestra en la Figura 4. Este módulo está relacionado directamente con la interacción entre las moléculas que se oponen al movimiento y deformación. En general, los geles de pectinas desesterificadas (PDE) presentaron valores de G' significativamente mayores en la var. GD que en la var. RChD, lo cual concuerda con el comportamiento observado por reología de alta deformación (Figura 3).

Se observó que a valores de $\mathrm{pH}$ de 3 y 4 , el gel muestra mayor elasticidad; sin embargo, a $\mathrm{pH}$ neutro $(\mathrm{pH} 7)$ disminuyó la $G^{\prime}$ de los geles de pectina de ambas fuentes, lo cual podría estar relacionado con un menor número de cargas en la molécula que afecta la interacción iónica con el calcio. Sin embargo, al aumentar el pH a 9 y 12 también aumentan los valores de G' de los geles, que alcanzan sus valores máximos a pH = 12 con 170 y 102 Pa para las vars. GD y RChD, respectivamente. Este aumento también podría estar relacionado con una desesterificación adicional de los grupos metilo en la cadena del polímero, y así deja disponibles los grupos carboxilo. En este tema también se requiere mayor investigación para generar evidencia concluyente en este respecto.

A pesar de que las comparaciones se realizaron entre variedades, es evidente que las pectinas de ambas variedades muestran un comportamiento alternante a cada valor de $\mathrm{pH}$. Específicamente, a $\mathrm{pH} 5$ parecería que los carboxilos libres tienen un estado de ionización que mejora su accesibilidad para interactuar con el calcio en el sistema, lo que no ocurre a otros valores de $\mathrm{pH}$ cercanos. Este comporta- miento concuerda con lo observado anteriormente para G", y confirma claramente una utilidad práctica. Las pectinas aquí probadas podrían interactuar eficazmente para estabilizar fases, mejorar textura, y como emulsificantes, toda vez que tales funciones se derivan de la interacción molecular del polímero con otros componentes de un alimento.

\section{CONCLUSIONES}

Los resultados de esta investigación sugieren fuertemente que las manzanas inmaduras de raleo pueden ser una buena fuente para la extracción de pectinas. Bajo las condiciones de extracción y desesterificacion aquí utilizadas, las pectinas presentan altos valores de peso molecular viscosimétrico y de viscosidad intrínseca. La desesterificación permite obtener pectinas de bajo grado de metoxilación, capaces de formar geles iónicos firmes.

La pectina desesterificada de manzana de raleo presenta propiedades físico-químicas y gelificantes muy cercanas a pectinas comerciales de interés para las industrias alimentaria, farmacéutica o de cosméticos, entre otras. La utilización de manzana de raleo como subproducto del manejo de huertos de manzano puede ser una fuente alternativa de pectina, lo cual podría considerarse como una posibilidad para incrementar la rentabilidad de los huertos y el desarrollo de nuevos procesos de transformación para el sector agrícola.

\section{AGRADECIMIENTOS}

Los autores agradecen el apoyo brindado por el Fondo

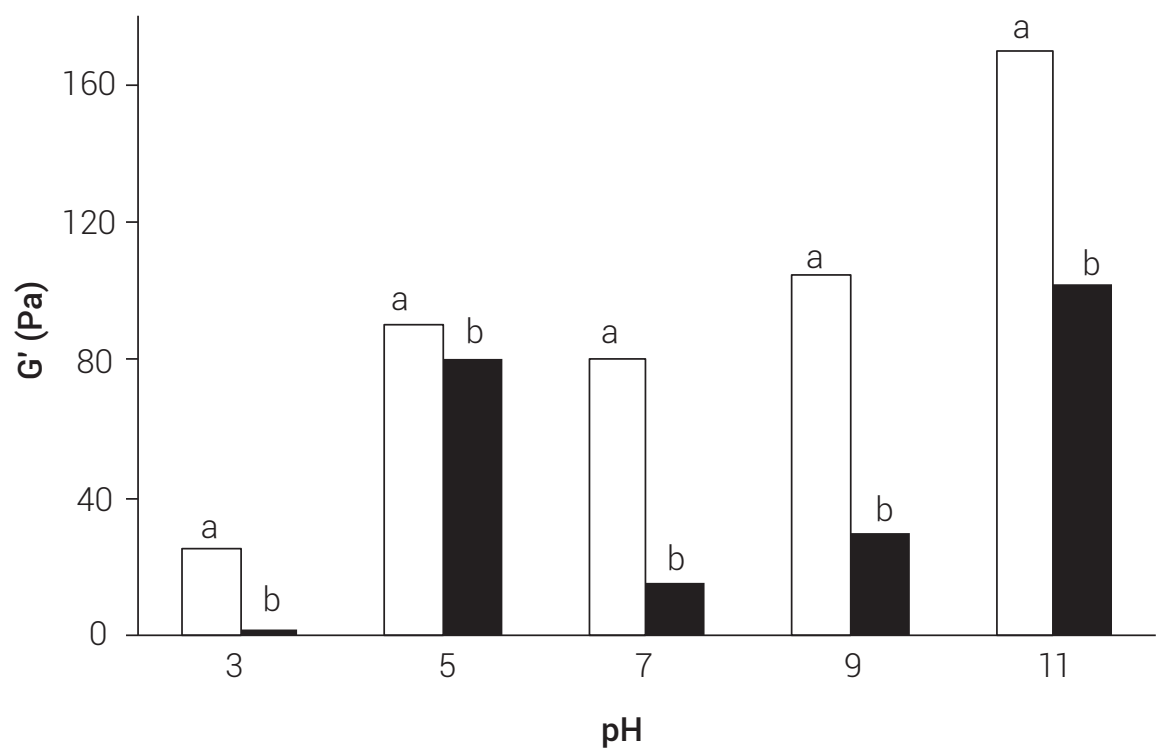

Figura 4. Comparación del módulo de almacenamiento $\left(G^{\prime}\right)$ del gel de pectina extraída de manzana de raleo a $2 \%(p / v)$ de las variedades Golden Delicious ( $n$ ) y RedChief Delicious (o), a distintos valores de $\mathrm{pH}$, entrecruzados con $\mathrm{CaCl}_{2}$ a $0.8 \%$ $(\mathrm{p} / \mathrm{v})$. Para un mismo valor de $\mathrm{pH}$, las medias con la misma letra son estadísticamente iguales (Tukey, 0.05). 
Proyectos y Fortalecimiento de Redes Temáticas CONACYT de Investigación formadas en 2009 (clave 194790).

\section{BIBLIOGRAFÍA}

Braccini I. and S. Pérez (2001) Molecular basis of $\mathrm{Ca}^{2+}$-induced gelation in alginates and pectins: The Egg-Box model revisited. Biomacromolecules 2:1089-1096.

Bradford M. M. (1976) A rapid and sensitive method for the quantification of microgram quantities of protein utilizing the principle of protein-dye binding. Analytical Biochemistry 72:248-254.

Bunzel M., J. Ralph and H. Steinhart (2009) Association of non-starch polysaccharides and ferulic acid in grain amaranth (Amaranthus caudatus I.) dietary fiber. Molecular Nutrition and Food Research 49:551-559.

Capel F., T. Nicolai, D. Durand, P. Boulenguer and V. Langendorff (2006) Calcium and acid induced gelation of (amidated) low methoxyl pectin. Food Hydrocolloids 20:901-907.

Carvajal-Millan E., B. Guigliarelli, V. Belle, X. Rouau and V. Micard (2005) Storage stability of arabinoxylan gels. Carbohydrate Polymers 59:181-188

Cindrić I. J., M. Kunštić, M. Zeiner, G. Stingeder and G. Rusak (2011) Sample preparation methods for the determination of the antioxidative capacity of apple juices. Croatica Chemica Acta 84:435-438.

Costa F. G. P., C. C. Goulart, D. F. Figueiredo, C. F. S. Oliveria and J. H. V. Silva (2008) Economic and environmental impact of using exogenous enzymes on poultry feeding. International Journal of Poultry Science 7:311-314.

Einhorn-Stoll U., H. Kunzek and G. Dongowski (2007) Thermal analysis of chemically and mechanically modified pectins. Food Hydrocolloids 21:1101-1112.

Fishman M., D. Gillespie, S. Sondney and Y. S. El-Atawy (1991) Intrinsic viscosity and molecular weight of pectin components. Carbohydrate Research 215:91-104.

Francini A. and L. Sebastiani (2013) Phenolic compounds in apple (Malus $x$ domestica Borkh.): compounds characterization and stability during postharvest and after processing. Antioxidants 2:181193.

Ishii T. (1997) O-Acetylated oligosaccharides from pectins of potato tuber cell walls. Plant Physiology 113:1265-1272.

Lootens D., F. Capel, D. Duran, T. Nicolai, P. Boulenguer and V. Langendorff (2003) Influence of $\mathrm{pH}, \mathrm{Ca}$ concentration, temperature and amidation on the gelation of low methoxyl pectin. Food Hydrocolloids 17:237-244.

Marcon M. V., L. C. Vriesmann, G. Wosiacki, E. Beleski-Carneiro and C. L. 0. Petkowicz (2005) Pectins from apple pomace. Polimeros: Ciên- cia e Technologia 15:127-129.

May C. D. (1997) Pectins. In: Thickening and Gelling Agents for Food. A. Imeson (ed.). Spreenger-Science+Business Media, B.V. London, UK. pp:231-261.

Mead D. J. and R. M. Fouss (1942) Viscosities of solutions of polyvinyl chloride. Journal of the American Chemical Society 64:277-282.

Mollea C., R. Chiampo and R. Conti (2008) Extraction and characterization of pectins from cocoa husks: A preliminary study. Food Chemistry 107:1353-1356.

Monsoor M. A., U. Kalapathy and A. Proctor (2001) Determination of polygalacturonic acid content in pectin extracts by diffuse reflectance Fourier transform infrared spectroscopy. Food Chemistry 74:233-238.

Powell D. A., E. R. Morris, M. J. Gidley and D. A. Rees (1982) Conformations and interactions of pectins: II. Influence of residue sequence on chain association in calcium pectate gels. Journal of Molecular Biology 155:517-531.

Rascón-Chu A., A.L. Martínez-López, E. Carvajal-Millán, N. Ponce de LeónRenova, J.A. Márquez-Escalante and A. Romo-Chacón (2009) Pectin from low quality 'Golden Delicious' apples: Composition and gelling capability. Food Chemistry 116:101-103.

Renard C. and M. C. Jarvis (1999) Acetylation and methylation of homogalacturonans 2: Effect on ion-binding properties and conformations. Carbohydrate Polymers 39:209-216.

Rinaldo M. (1996) Physicochemical properties of pectins in solution and gel states. In: Pectins and Pectinases. J. Visser and A. G. J. Voragen (eds.). Elsevier Science B.V. Amsterdam, The Netherlands. pp:21-33

SAS (1994) SAS/STAT User's Guide. Release 6.08 Version.SAS Institute Inc. Cary, NC, USA. 705 p.

Saulnier L. and J-F. Thibault (1999) Ferulic acid and diferulic acids as components of sugar-beet pectins and maize bran heteroxylans. Journal of the Science of Food and Agriculture 79: 396402.

Siew C. K. and P. A. Williams (2008) Role of protein and ferulic acid in the emulsification properties of sugar beet pectin. Journal of Agricultural and Food Chemistry 56:4164-4171.

Ström A. and M. A. K. Williams (2003) Controlled calcium release in the absence and presence of ion-binding polymer. Journal of Physical Chemistry B 107:10995-10999.

Urias-Orona V., A. Rascón-Chu, A., J. Lizardi-Mendoza, E. Carvajal-Millán, A. A. Gardea and B. Ramírez-Wong (2010) A novel pectin material: extraction, characterization and gelling properties. International Journal of Molecular Sciences 11:3686-3695.

Yoo S. H., M. L. Fishman, A. T. Hotchkiss and H. G. Lee (2006) Viscometric behavior of high-methoxy and low-methoxy pectin solutions. Food Hydrocolloids 20:62-67. 